\title{
Cephalic Tetanus Presenting With Trismus in an Intravenous Drug Abuser
}

\author{
Mehrdad Haghighi ${ }^{1}$; Amirhossein Moghtader Mojdehi ${ }^{1}$; Shiva Faridi ${ }^{1, *}$ \\ ${ }^{1}$ Department of Infectious Diseases, Imam Hossein Teaching and Medical Hospital, Shahid Beheshti University of Medical Sciences, Tehran, IR Iran \\ *Corresponding author: Shiva Faridi, Department of Infectious Diseases, Imam Hossein Teaching and Medical Hospital, Shahid Beheshti University of Medical Sciences, Tehran, IR \\ Iran. Tel: +98-9185338640, E-mail: shiva3faridy@yahoo.com
}

Received: September 7, 2014; Revised: January 18, 2015; Accepted: January 20, 2015

\begin{abstract}
Introduction: Cephalic tetanus is a rare form of tetanus, defined as trismus and cranial nerve palsy. It has a mortality rate of 15 - $30 \%$. Case Presentation: The purpose of this case report was to present a case of cephalic tetanus with trismus, a rare form of tetanus, in an intravenous drug abuser. Timely diagnosis and management prevented progression into the generalized form and made the patient needless of invasive procedures.

Conclusions: Two main risk factors for getting tetanus include not being immunized against it and decrement in immunity in the elderly. Catch up vaccination programs should be carried out to cover the previously unimmunized individuals, especially in high-risk groups such as intravenous drug abusers. Booster doses of tetanus and diphtheria toxoids vaccine(Td) for the elderly should be considered.
\end{abstract}

Keywords:Trismus; Tetanus; Drug Abuser

\section{Introduction}

Tetanus is an acute infectious disease, characterized by skeletal muscle spasm and autonomic nervous system disturbance. Tetanus is caused by the tetanospasmin neurotoxin, produced by Clostridium tetani. It is a vaccine-preventable disease; thus, it commonly occurs in developing countries where the vaccination coverage rate is low (1). Injection drug abuse places patients at risk for tetanus (2). Clinically, tetanus appears in four forms of generalized, neonatal, localized and cephalic. Generalized tetanus accounts for $80 \%$ of cases (3), while cephalic tetanus is rare, accounting for only 1-3\% of all tetanus cases (4). Cephalic tetanus is defined as trismus plus paralysis of one or more cranial nerves. Here, a case of cephalic tetanus in a 34-year-old intravenous drug abuser male, not immunized against tetanus, is presented. Treatment involved debridement of wounds, administration of penicillin and tetanus immune-globulin, aggressive supportive care, and initiation of active immunization (4).

\section{Case Presentation}

A 34-year-old male referred to the emergency room, complaining of inability to move his jaw, which lasted for 20 minutes. Taking his history showed that he had experienced the same symptoms three times during the two days before the admission. During these attacks, he was completely conscious and had sialorrhea as well. In addition, he felt a severe pain in masticatory muscles, especially in the temporalis muscle. He remarked that during the attacks, his tongue flattened out of his mouth and he had to push it inside passively. He also had difficulty speaking postictally.

The patient was unemployed and an intravenous drug abuser. He had no remarkable past medical history other than severe dental caries. He had not completed the routine immunization program from childhood; notably, he had had no tetanus shots. He has smoked for the last five years and used illicit drugs such as heroin, crack and methamphetamine for the last three years. He has not attended the military service.

Alprazolam is the only drug that the patient said he used, but he was not reliable. He had dysphagia, but no dyspnea. He did not have a history of penetrating trauma to the head or any other body part. On physical exam, he was pale but conscious and his vital signs were within the normal range.

He had a clear site of an abscess, a punched out ulcer on the left mandibular area of his face from 40 days before the admission, which had drained spontaneously a month after its appearance. No lesion was seen in his mouth. He had no palpable lymphadenopathy.

The neurological examination was completely normal. The examination of the cardiovascular and respiratory systems was also normal. There was no abdominal muscle rigidity. Based on clinical grounds and the initial findings, we considered four differential diagnoses: cephalic tetanus, oropharyngeal abscess, strychnine poisoning, and acute dystonia.

The performed head and neck CT scans revealed no current dental or oropharyngeal abscess. Ultrasonography of 
the mandibular soft tissue revealed no sign of collection or mass. The patient did not have a history of neuroleptic or antidopaminergic drugs, therefore pushing acute dystonia out of the differential diagnosis list. Strychnine was not detected in the patient's serum, which spared cephalic tetanus to be the final diagnosis.

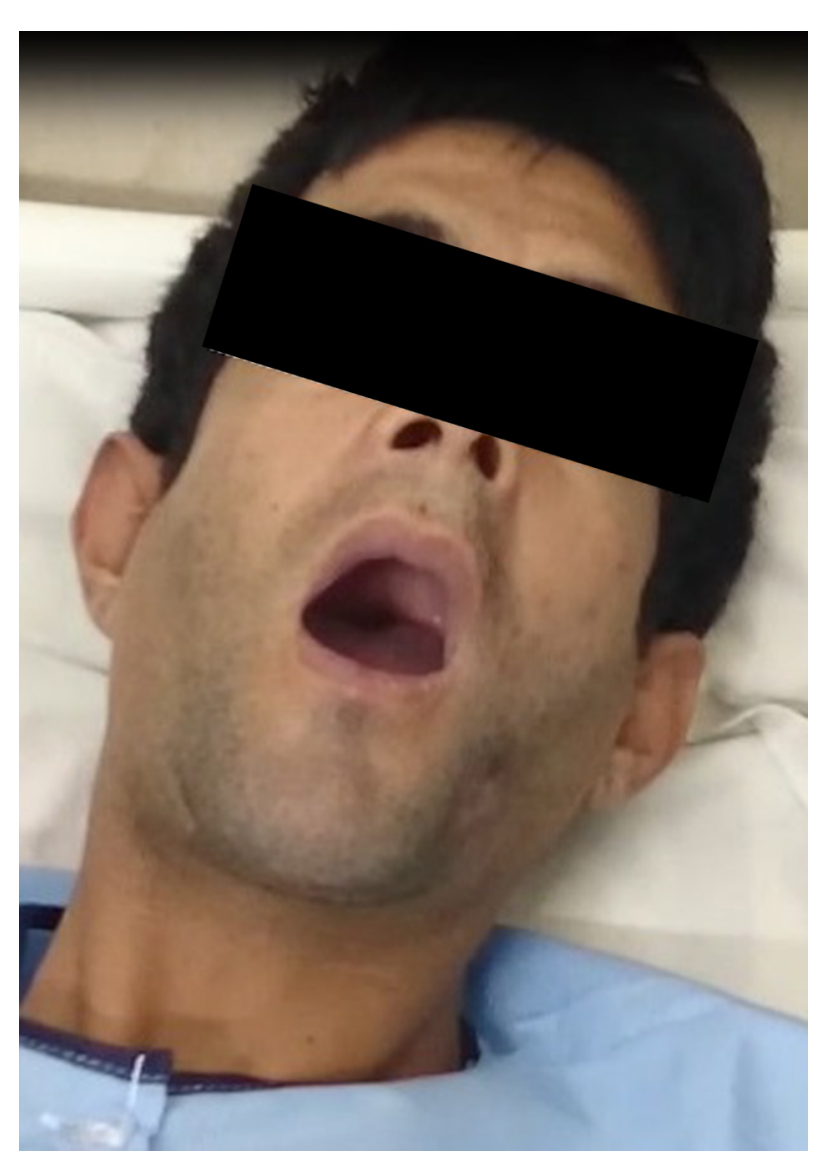

Figure 1. Masseter Rigidity Resulting in the Inability to Move the Jaw, Called Trismus

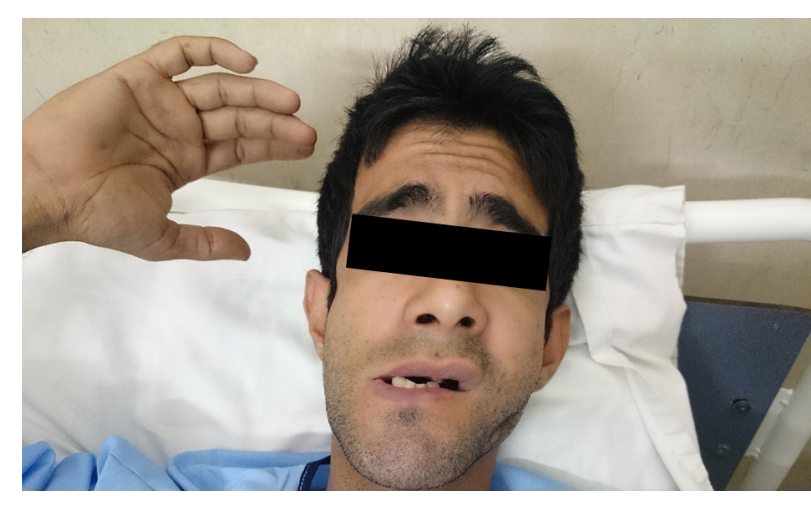

Figure 2. Facial Paresis, Resultant Grimace and the Bilateral Blepharospasm
The lab workout revealed no abnormal findings. The blood culture showed no growth. Since infective endocarditis is a serious complication in intravenous drug abusers (5), echocardiography was carried out, which revealed no abnormal findings.

In the emergency room, he received metronidazole intravenously and midazolam; as a result, the trismus resolved.

He was hospitalized at the infectious ward for two weeks. He was also in the intensive care unit admission list, but was not transferred because there was no vacancy. During the hospitalization, he had another attack. The trismus and the grimace were readily notable (Figures 1 and 2).

Tetabulin and tetanus and diphtheria toxoids (Td) vaccine were administered stat in the emergency room. During the attacks, the patient received $3 \mathrm{mg}$ of midazolam. He received another $\mathrm{Td}$ vaccine ampule when leaving and another is going to be injected six weeks after the second injection. The antibiotic therapy included penicillin ampule every four hours and metronidazole ampule every six hours. It did not progress into the generalized form and he was treated without any complications.

\section{Discussion}

Cephalic tetanus accounts for $1-3 \%$ of the total number of reported cases of tetanus and has a mortality rate of $15-30 \%$. The incubation period is 1 to 14 days and approximately two thirds of the cases progress to generalized tetanus (1).

Tetanus is diagnosed based on clinical grounds; thus, it is very important to recognize its presenting symptoms. Clinicians might be unfamiliar with the presentations of cephalic tetanus due to its infrequency; as a result, later mismanagement imperils the patient's life. Delay in diagnosis and management worsens the prognosis; that way, invasive procedures such as tracheostomy might be indicated, imposing consequent complications on the patient (6). Among the reported symptoms are trismus (7-10), dysphagia $(7,8,11)$, cranial nerve palsy (11), signs such as blepharospasm $(7,12)$, facial paresis (8), ptosis (8), and vertigo (10).

Two main risk factors for getting tetanus are: not being immunized against it and decrement of immunity in the elderly. History of a recent penetrating trauma is a key factor in diagnosis; but, the intravenous drug abuse, as in this case, places the patient at a higher risk as well. The location of injury and its distance from the head used to be considered as important factors in the development of cephalic tetanus, but injuries far from the head can also cause it (7).

Preventing tetanus should be a high priority for all primary care physicians (13). Catch-up vaccination programs should be carried out to cover the previously unimmunized individuals, especially in high-risk groups such as intravenous drug abusers. Booster doses of Td for the elderly should be considered. 


\section{Authors' Contributions}

Mehrdad Haghighi introduced the case and the idea of reporting it. Amirhossein Moghtader Mojdehi contributed to the acquisition and interpretation of data. Shiva Faridi contributed to the acquisition and analysis of data as well as drafting and revision of the manuscript.

\section{References}

1. Edsall G. Editorial: The inexcusable disease.JAMA.1976;235(1):62-3.

2. Beeching NJ, Crowcroft NS. Tetanus in injecting drug users. BMJ. 2005;330(7485):208-9.

3. Edlich RF, Hill LG, Mahler CA, Cox MJ, Becker DG, Horowitz JH, et al. Management and prevention of tetanus. J Long Term Eff Med Implants. 2003;13(3):139-54.

4. Jagoda A, Riggio S, Burguieres T. Cephalic tetanus: a case report and review of the literature. Am J Emerg Med.1988;6(2):128-30.

5. Soudbakhsh A, Hagiabdolbaghi M, Bagherian H, Yussefnejad O. Comparing clinical and laboratory findings of infective endocar- ditis among intravenous drug users and non-drug users. Iran J Clin Infec Dis. 2007;2(4):181-4.

6. Schon F, O'Dowd L, White J, Begg N. Tetanus: delay in diagnosis in England and Wales. J Neurol Neurosurg Psychiatry. 1994;57(8):1006-7.

7. Kwon JC, Park Y, Han ZA, Song JE, Park HS. Trismus in cephalic tetanus from a foot injury. Korean J Intern Med. 2013;28(1):121.

8. Seo DH, Cho DK, Kwon HC, Kim TU. A case of cephalic tetanus with unilateral ptosis and facial palsy. Ann Rehabil Med.2012;36(1):167-70.

9. Guyennet E, Guyomard JL, Barnay E, Jegoux F, Charlin JF. Cephalic Tetanus from Penetrating Orbital Wound. Case Rep Med. 2009;2009.

10. Kagoya R, Iwasaki S, Chihara Y, Ushio M, Tsuji S, Murofushi T, et al. Cephalic tetanus presenting as acute vertigo with bilateral vestibulopathy. Acta Otolaryngol. 2011;131(3):334-6.

11. Park DM. Cranial nerve palsies in tetanus: cephalic tetanus. J Neurol Neurosurg Psychiatry. 1970;33(2):212-5.

12. Liu CY, Liao KK, Fuh JL, Wang PN, Shan DE, Tsai CP. Unilateral blepharospasm as an early sign of cephalic tetanus. Mov Disord. 2009;24(7):1094-5.

13. Bleck TP. Tetanus: pathophysiology, management, and prophylaxis. Dis Mon.1991;37(9):545-603. 